\title{
New defect detection approach using near electromagnetic field probing of high density PCBAs
}

\author{
N. El Belghiti Alaoui ${ }^{\mathrm{a}, \mathrm{b},{ }^{*}}$, A. Boyer ${ }^{\mathrm{a}}$, P. Tounsi ${ }^{\mathrm{a}}$, A. Viard ${ }^{\mathrm{b}}$ \\ a Laboratoire CNRS, LAAS, 7 avenue du colonel Roche, Univ. de Toulouse, INSA, LAAS, F-31400 \\ ${ }^{\mathrm{b}}$ ACTIA Automotive, 5 Rue Jorge Semprun, 31400, France
}

\begin{abstract}
With the density increase of today's printed circuit board assemblies (PCBA), the electronic fault detection methods reached their limits. In the same time the requirements of high reliability and robustness are greater. Industrials are obliged to find better-adapted test methods. Current test methods must be rethought to include a large panel of physical phenomena that can be used to detect electrical defects of components, absence, wrong value, and shorts at component level on the board under test (BUT).

We will present the possibility of using electromagnetic signature to diagnose faulty components contactlessly. The technique consists in using magnetic field probes, which detect the field distribution over powered sensitive components. Reference EM signatures are extracted from a fault-free circuit, which will be compared to those extracted from a sample PCBA in which we introduced a component level defect by removing or changing the value of critical components to evaluate the relevance of the method.
\end{abstract}

Keywords - Testability, Accessibility, PCB assembly production test, near field probes, magnetic sensors, giant magnetoresistance, In-circuit test, Design for Testability, contactless testing, High density PCBA testing,

\section{Introduction}

During the assembly process of printed circuit boards, defects such as wrong value components, missing components, unwanted open circuits or short circuits may appear. Manufacturers continually look for faster, more accurate and more economical ways to identify this kind of defects. That's why performing automated testing of dense populated printed circuit boards is a mandatory and cost effective solution to ensure manufacturing quality control.

Testing today's populated PCBs is becoming increasingly challenging and more expensive as the use of small size surface mounted devices (SMD) is becoming predominant. The emergence of new technologies as High-Density Interconnect (HDI), embedded chips and Sequential Build-Up (SBU) circuit boards will even further increase the challenge for the test business.

Conventional techniques for automated PCBA testing involve applying signals through a number of test pins and measuring the output signals on the other test pins. Functional testing can be performed by energizing the PCBA, applying a predetermined number of input signals, and determining whether the proper output signals are generated by the circuitry on the BUT [1]. Alternatively, for a high volume manufacturing (HVM) process, a PCBA is tested primarily on a "bed-of-nails" fixture called in-circuit tester (ICT) that comprises pins called "nails" which directly contact the metallic traces on the BUT so that selected input signals may be applied at various test points (TP) on the PCB, and corresponding output signals can be measured on other TPs. This requires several physical TPs on the PCB traces which can compromise the integrity of the tested signals.

This widely used classical technique requires tight mechanical tolerances for the board layout, easily accessible test points and restricts the frequency band at which a board can be tested [2], which cannot be afforded anymore on a state of the art PCBA. Starting from this need, the idea of taking advantage of the HVM nature of the ICT and trying to upgrade it with contactless probes to meet current test challenges have come. In this paper, we present a new testing approach using EM near field sensors (NFS) to test populated PCBs.

The principle of the proposed method is given in order to understand its large scale application. To this aim and to prove the effectiveness of the method, we chose a DC/DC buck converter module as a case study. Simulations of value defect scenarios have been carried out on Cadence Orcad and validated by measurements on the module in which we introduced controlled value defects on the input decoupling 
ceramic capacitors. First results to validate the principle and perspectives for future work are reported.

\section{Description of the proposed testing approach}

To improve the effectiveness and accessibility of current ICTs and gain accessibility to components that could not be tested without placing numerous test points, we propose in this article a new test approach based on EM inspection using near field sensors as an upgrade to the classical In-circuit testers. With magnetic field measurements above PCBA components, contactless information about current distribution can be obtained without access limitation on the PCBA surface.

\subsection{Principle}

The principle of this method (see Fig.1) is to measure the EM near field distribution directly over the center of a powered component contactlessly and compare the measured electromagnetic signature (EMS) to a database of correct signatures preestablished on a fault-free PCBA. The measured electric and magnetic fields (EMF) are generated by the distribution of charges and currents respectively in the components of the BUT. Accurate and repeatable measurements of these fields produce a specific time and frequency domain signature for each critical component, which can be extended to a complete operational block, and then to the complete board in a further application. Such signatures are then compared to a pre-established non-faulty signature pattern of the same type of board to determine whether the BUT is faulty or not and pinpoint exactly where the faulty component is located knowing the position of the NFS on the board.

Thus firstly, the board or the operational block of the board is powered and operates normally. The electromagnetic near-field distribution generated by every "critical" component on the block is then detected using non-contact NFS mounted directly over these components in a bed-of-nails structure. The registered signature specific to the component and the conditions of the excitation of the electronic block is registered and subsequently compared with a sample signature of the same block in a non-faulty board, which was registered in the same excitation conditions to determine whether the response is in conformance with the known reference.

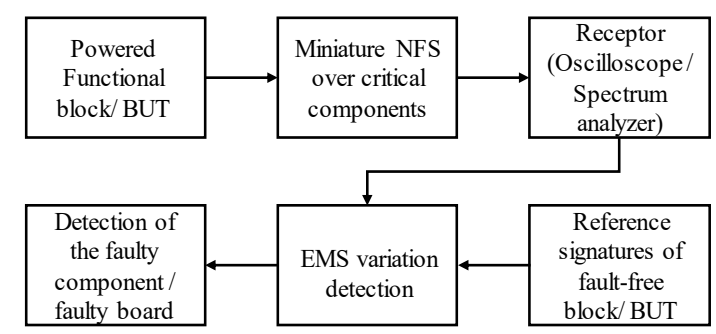

Fig. 1. Principle of the near field probing test approach

\subsection{Types of detected assembly defects}

The defects that can be detected with this method are at component level. In other words, starting from the hypothesis that the bare PCB is fault free guaranteed from the printed circuit manufacturer, or had already been tested and certified non-faulty. We can detect assembly defects over critical components: presence, polarity for components that exhibits field change when mounted in reverse polarizations, value, a wrong package that can change the height of the component, overstressed/overheating components and solder defect (open and shorts). These components must be carefully chosen in advance in order to establish design for testability rules (DFT) to minimize the probe count and maximize fault detection on a functional block level.

\section{Case study: DC/DC buck converter}

To validate our approach, we chose a DC/DC converter because of the important transient currents crossing the critical components when the module is powered. Components such as input/output filtering capacitors, MOSFETS and inductors radiate a significant high frequency magnetic field in the near field region due to the large transient currents crossing them. The currents and the induced magnetic fields are related to component values, package and mounting. From the analysis of measured magnetic fields, the presence and the location of assembly defects or wrong components can be detected.

This case represents a scenario of testing a $\mathrm{DC} / \mathrm{DC}$ converter module in a power management block of an industrial high density PCBA using the $\mathrm{MF}$ radiated from its critical components. Using a commercial near field probe (NFP) and a GMR sensor, the EM signature of critical components that have a high frequency transient current passing through them, as described above, is registered to establish a sample signature of each component.

We chose an off-the shelf (OTS) evaluation board of a synchronous DC/DC buck converter module with a fully integrated controller to run tests. The powering conditions for test are as mentioned in Table 1.

Table 1

Powering conditions for test

\begin{tabular}{ll}
\hline $\mathrm{V}_{\text {in }}$ & $20 \mathrm{~V}$ \\
$\mathrm{~V}_{\text {out }}$ & $12 \mathrm{~V}$ \\
ILoad $_{\text {Lorequency }}$ & $3 \mathrm{~A}$ \\
Freque & $250 \mathrm{kHz}$ \\
\hline
\end{tabular}

\subsection{Simulated defect scenarios:}

The defect scenario used to validate this approach is the detection of a wrong value of an input decoupling capacitor, output capacitor and filtering inductor.

The approach is tested initially in simulation, and then validated in measurement. 


\subsubsection{Test procedure: Simulation}

We modeled the DC/DC buck converter evaluation board on Cadence-Orcad using the pspice model of the controller given by the manufacturer (see Fig.2). Estimated values of parasitic elements of each critical component were used to give a more accurate simulation result. Then, we ran multiple parametric simulations of the value of each input capacitor with four different values (see Table 2) to evaluate how the derivative of the current in each input capacitor reacting to a change of value of an input capacitor and the induced variation over the other capacitors that have correct values. The derivative of the current in a component represents the image of the magnetic field measured with a commercial NFP over this specific component.

Table 2.

Input capacitor values in simulation and experiments

\begin{tabular}{lll}
\hline $\begin{array}{l}\text { Input } \\
\text { capacitors }\end{array}$ & $\begin{array}{l}\text { Correct } \\
\text { value }(\mu \mathrm{F})\end{array}$ & $\begin{array}{l}\text { Incorrect } \\
\text { values }(\mu \mathrm{F})\end{array}$ \\
\hline $\mathrm{C} 8$ & $2.2 \mu \mathrm{F}$ & $1,1.5,3.3,15$ \\
$\mathrm{C} 9$ & $2.2 \mu \mathrm{F}$ & $1,1.5,3.3,15$ \\
$\mathrm{C} 10$ & $2.2 \mu \mathrm{F}$ & $1,1.5,3.3,15$ \\
$\mathrm{C} 11$ & $2.2 \mu \mathrm{F}$ & $1,1.5,3.3,15$ \\
\hline
\end{tabular}

\subsubsection{Test procedure: Experimentation}

We reproduced the same scenario described in the test procedure simulation with the same values on the evaluation DC/DC converter module. We changed the value of each input capacitor on the board several times by soldering and removing a different value capacitor for every input ceramic capacitor (C8,C9,C10,C11), and we collected the time domain signatures over each capacitor using an oscilloscope for each of the four values evaluated. Collected signatures of each capacitor were analyzed to evaluate their variance compared to the reference signatures.

\subsubsection{Test bench description}

\section{- Near field probes:}

Measuring the time domain $\mathrm{H}$-field signatures over the powered (see Table 1) DC/DC buckconverter was carried out using a commercial mini (resolution $<1 \mathrm{~mm}$ ) Near-Field probe (LANGER RFR 0.3-3) (see Fig.4) which measures lateral magnetic fields over the components in the range from $30 \mathrm{MHz}$ to $3 \mathrm{GHz}$. The probe was directly connected to a digital oscilloscope with $50 \Omega$ input impedance. The NFP was then freely and accurately moved over every input capacitor in near-field region at a $2 \mathrm{~mm}$ distance of the center of the component using an automatic computer controlled scan table with a distance precision of $25 \mu \mathrm{m}$ (see Fig. 3).

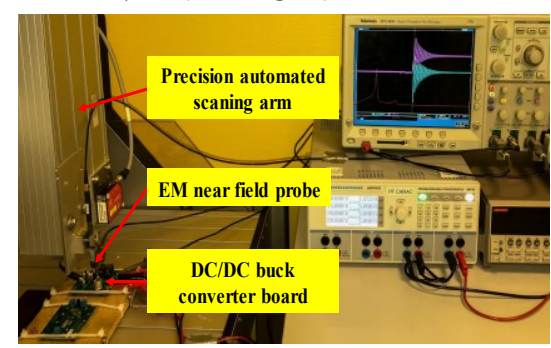

Fig. 3. Test bench set-up

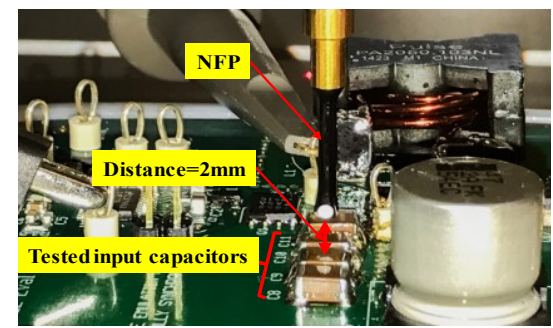

Fig. 4. Magnetic NFP and the probed components

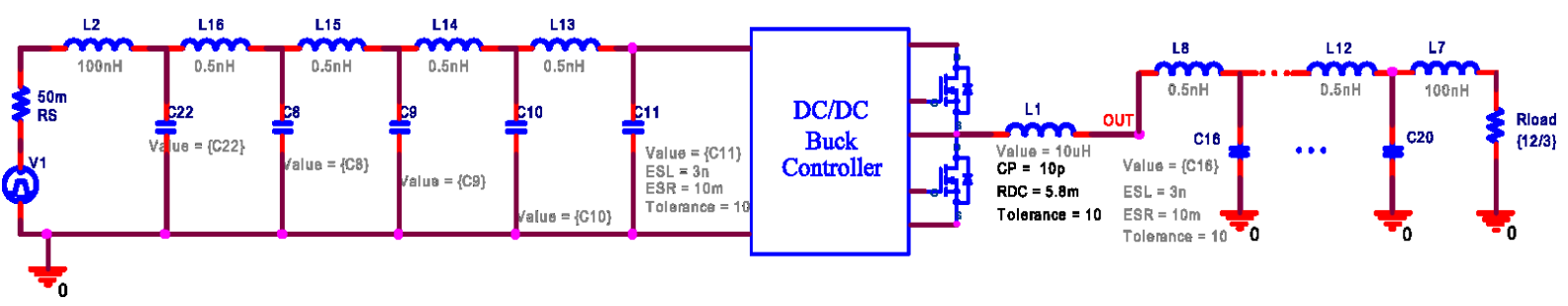

Fig. 2. Simplified schematic of simulation

\section{- GMR Sensors}

Near field probes have a high sensitivity when measuring high frequency fields, on the other hand, low frequency fields are not detected. To compensate the lack of sensitivity in low frequency measurements, another type of magnetic-field sensor is used: GMR sensor.

The giant magnetoresistance effect (GMR) effect discovered in 1988 [3] is related to field dependent changes in resistance that can be observed in thin-film ferromagnetic/non-magnetic metallic multilayers. The term GMR was coined due to the large change of resistance ( 10 to $20 \%$ ) of the thin-film materials. GMR sensors have taken an important role due to their small size, high signal level, high sensitivity, large frequency response and low cost [4].

Contrary to the NFP, this sensor provides better sensitivity for relatively low frequency magnetic fields (up to $1 \mathrm{MHz}$ ) [5]. In a DC/DC converter, such low frequency magnetic field dominates above the output inductor. The low frequency current ripple crossing the inductor can be measured with a GMR sensor to detect any variation of the inductance.

In this study, a commercial multilayer GMR sensor with a sensitivity of $5.4 \mathrm{mV} / \mathrm{V} / \mathrm{A}$ for frequencies up to $100 \mathrm{KHz}$ from NVE spintronic is used. 
A dynamic characterization of the sensor shows a sensitivity drop of $3.16 \mathrm{mV} / \mathrm{V} / \mathrm{A}$ per decade for greater frequencies.

At around $400 \mathrm{KHz}$, the switching frequency of this converter, the sensitivity of the sensor $\left(S_{\text {dynamic }}\right)$ is estimated at $3.8 \mathrm{mV} / \mathrm{V} / \mathrm{A}$.

\section{- Test conditions :}

We used the commercial GMR sensor to test a 2525 SMD inductor mounted on a different DC/DC Buck converter evaluation module (see Table 3).

The measurements were taken at a distance of $2 \mathrm{~mm}$ from the surface of the inductor for four different values (see Fig. 5). The converter was powered on, and the output load current was constant at $3 \mathrm{~A}$.

Table 3

Test conditions

\begin{tabular}{ll}
\hline $\mathrm{V}_{\text {in_DC/DC }}$ & $10 \mathrm{~V}$ \\
$\mathrm{~V}_{\text {out_DC/DC }}$ & $1.2 \mathrm{~V}$ \\
$\mathrm{~V}_{\text {supply_GMR }}$ & $20 \mathrm{~V}$ \\
$\mathrm{~F}_{\text {sw }}$ & $400 \mathrm{KHz}$ \\
ILoad & $3 \mathrm{~A}$ \\
Inductor reference value & $\mathrm{L}=0,47 \mu \mathrm{H}$ \\
\hline Inductor wrong values & $\mathrm{L}=0,22 \mu \mathrm{H}$ \\
to be detected & $\mathrm{L}=82 \mu \mathrm{H}$ \\
& $\mathrm{L}=1,5 \mu \mathrm{H}$ \\
\hline
\end{tabular}

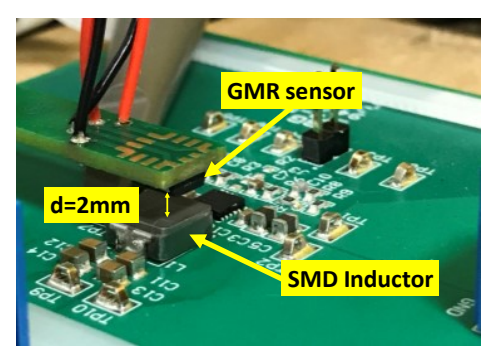

Fig. 5. Image of the GMR sensor and the measured SMD inductor

\section{Simulation and experimental results: \\ 4.1. Above the input decoupling capacitors \\ 4.1.1 Reference signatures:}

The reference signature is the magnetic field captured by the NFP over each input capacitor when it has a correct value.

Figure 6 shows that only around a specific frequency we obtain the same significant signature in simulation and measurement over input capacitors C8, C9, C10, and C11.

The noticeable difference in amplitude and frequency scales between simulation (dashed lines) and measurement (solid lines) is due to assumptions made on the parasitic parameters of the components in the simulation model to be more considered in a future work. This is also due to the coupling parameter of the NFP that wasn't taken into account in the simulation model.

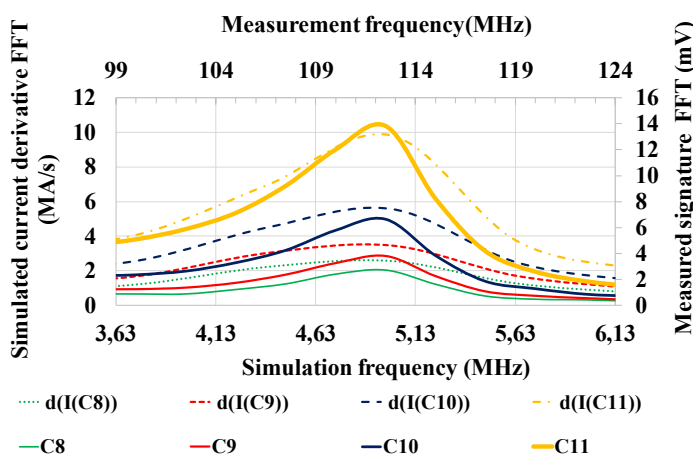

Fig. 6. Simulated current derivatives FFT of input capacitors (dashed lines), (vs) measured EM signatures FFT on the buck converter PCB

\subsubsection{Signatures with wrong values of the input capacitors:}

In concerns of conciseness, only the signatures measured over all input capacitors induced from the variation of the value of the input capacitor $\mathrm{C} 8$ will be presented in this section (see Fig. 7 and 8).

The dashed lines show the small signature variations of the unchanged input capacitors $(\mathrm{C} 9, \mathrm{C} 10$, $\mathrm{C} 11)$. Bold lines are the variation of $\mathrm{C} 8$ signatures when we varied its value. Results from simulation and measurements show that the magnetic signature of $\mathrm{C} 8$ varies significantly, while other capacitors signatures remain roughly unchanged.

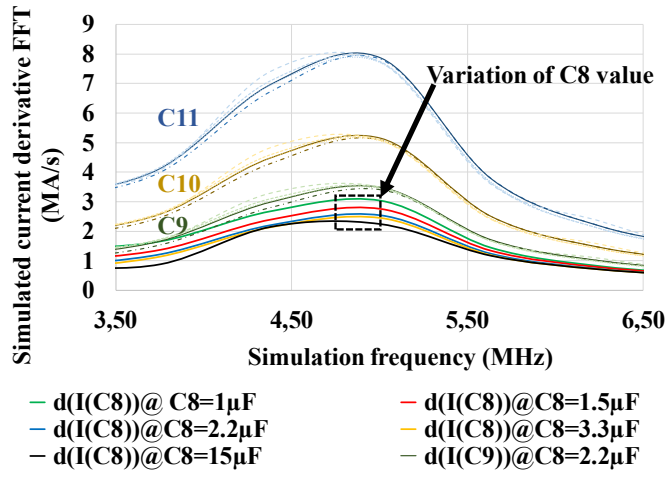

Fig. 7. Simulated current derivatives FFT of input capacitors when $\mathrm{C} 8$ varies

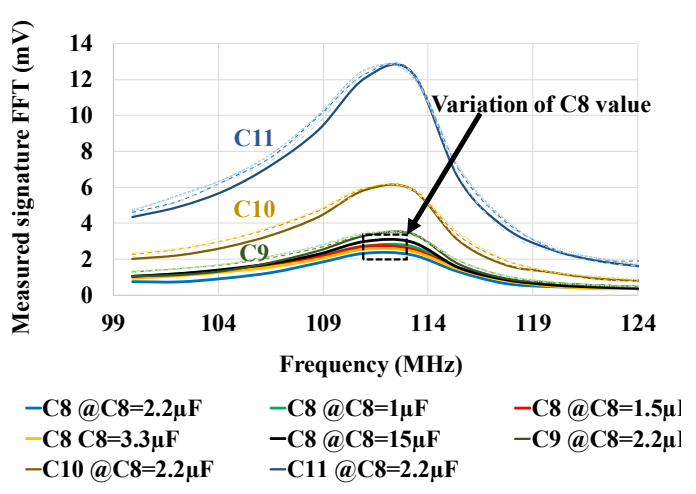

Fig. 8. Measured magnetic signatures FFT of the input capacitors when $\mathrm{C} 8$ varies 


\subsubsection{Comparison and analysis}

From the measurement results we can see that the capacitor value change induces a significant variation on the amplitude spectrum at around $110 \mathrm{MHz}$ of the EM signature of the capacitor being changed. This frequency is due to the resonance of the input capacitors with the parasitic elements of the switching stage of the converter, which depend on the board under test and the mounted devices.

This is still true for all input capacitors when we change their value. They all exhibit a significant variance of their signature FFT amplitude at around $110 \mathrm{MHz}$.

We summarized all simulation and measurment results of all changing capacitors $(\mathrm{C} 8, \mathrm{C} 9, \mathrm{C} 10, \mathrm{C} 11)$ in the graph below (see Fig. 9.a and 9.b), which shows that the most scattered signatures around the reference are those of the decoupling capacitor for which the value was changed. The dispersion of signatures here presented by the standard deviation to the reference signature remains lower for all value-unchanged capacitors.

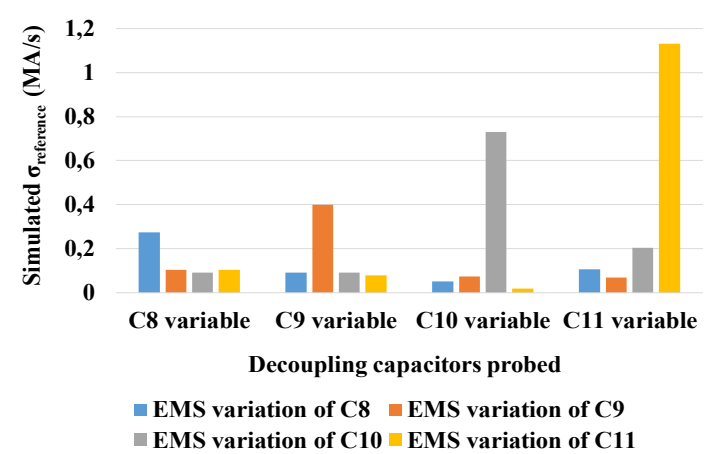

Fig. 9. a. Standard deviation of the simulated FFT amplitude of the magnetic signatures in each case where the value of one input capacitor is changed

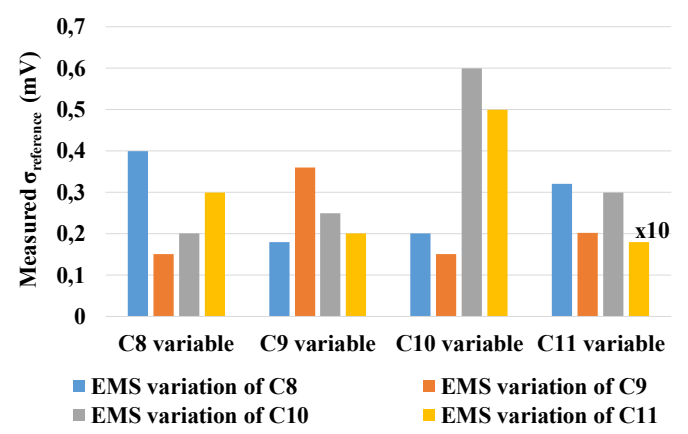

Fig. 9. b. Standard deviation of the measured FFT amplitude of the magnetic signatures in each case where the value of one input capacitor is changed separately

x10 The value of this deviation is $1,8 \mathrm{mV}$, it was divided by 10 to fit in the comparison graph.

We can see that there is a coherence between simulation and experimental results. The standard deviation of the value-changed capacitor is always the highest, which allows the detection of a capacitor with a wrong value through the comparison with a reference signature without the need of using test points.

\subsection{Above the output filter capacitors}

The output capacitance of a switching DC/DC converter is a vital part of the overall feedback system. The energy storage inductor and the output capacitors form a second-order low-pass filter. The output filter's inductor therefore limits the current slew rate. When the amount of current required by the load changes, the initial current deficit must be supplied by the output capacitors until the regulator can meet the load demand [6].

To measure magnetic field signatures over these output capacitors we need to emphasize on their effect by pushing them to provide a high transient current to the load.

To do so, we designed a load that provides a current step by switching the output current of the converter from $50 \mathrm{~mA}$ to $2.5 \mathrm{~A}$, with a rising time of $1 \mu \mathrm{s}$ and a falling time of $0.5 \mu \mathrm{s}$.

\subsubsection{Signatures with wrong values of the output capacitors}

Magnetic field signature measurements with NFP over each changing output capacitor (see Table 4) show clearly which capacitor's value is being changed. Only the signatures measured over the accessible top board capacitors (C16 and $\mathrm{C} 20)$ are here presented (see Fig. 10 and 11).

The dashed lines show the small signature variations of the unchanged output capacitors. Bold lines are signature variations of the changed output capacitor.

Table 4

Values used for the output capacitors

\begin{tabular}{lll}
\hline Input & Correct & Incorrect \\
capacitors & value $(\mu \mathrm{F})$ & values $(\mu \mathrm{F})$ \\
$\mathrm{C} 16$ & $47 \mu \mathrm{F}$ & $22,33,68$ \\
$\mathrm{C} 20$ & $22 \mu \mathrm{F}$ & $10,15,33$ \\
\hline
\end{tabular}

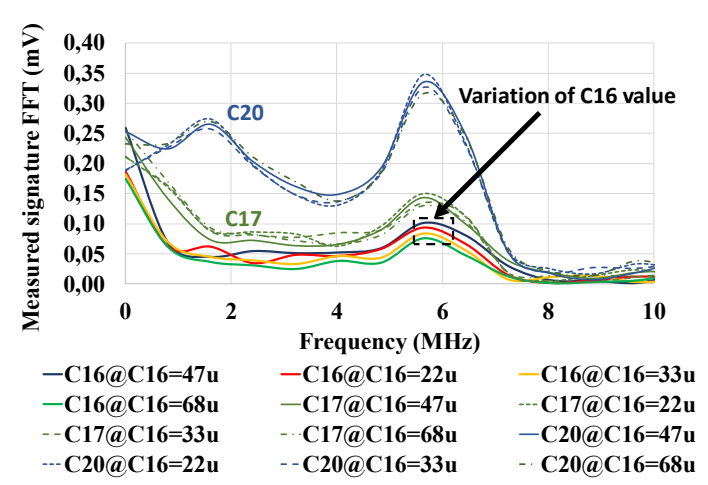

Fig. 10. Measured magnetic signatures FFT of the output capacitors when $\mathrm{C} 16$ varies 


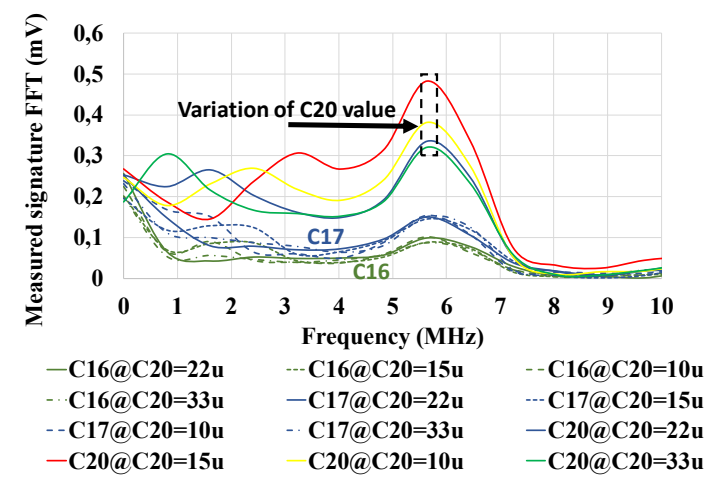

Fig. 11. Measured EM signatures FFT of the output capacitors when $\mathrm{C} 20$ varies

We can clearly distinguish the capacitor with a wrong value from the amplitude of its signature deviating from the reference one. This measured signature is the resonance of the loop composed of the output capacitors and the current step PCB parasitic elements (trace inductances and output load parasitic capacitances). In this particular case, we observed the resonance at $5.5 \mathrm{MHz}$.

\subsection{Above the output filter inductance using a GMR sensor}

The results presented below (see Fig. 12) show the possibility to detect variations of the value of the inductor using a GMR sensor. The peak-to-peak amplitude of the sensor's output voltage doubles as the value of the inductor is divided by 2 , which is coherent since the sensors output has a linear relationship with the AC magnetic field $(B)$ which is proportional to the current ripple $\left(\Delta I_{L}\right)$ in the inductor (see equation (1)).

$$
\begin{aligned}
& V_{\text {ou }_{G M R}}=S_{\text {dynamic }}(f) \times B\left(\Delta I_{L}\right) \\
& \Delta I_{L}=\frac{1}{F_{S W} X L} V_{\text {out }_{D C / D C}}\left(1-\frac{V_{\text {ou }} D C / D C}{V_{\text {in }}}\right)
\end{aligned}
$$

$F_{s w}$ is the converter's switching frequency and $L$ is the value of the filtering inductance. All measurements are in raw conditions, no shielding, filtering or amplification were used.

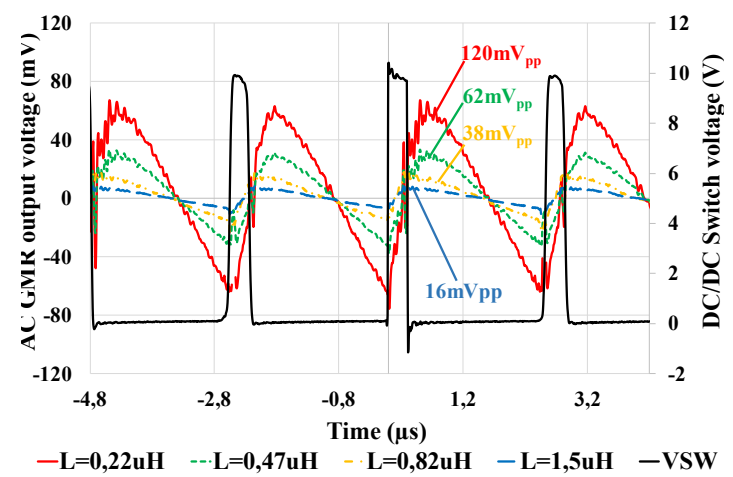

Fig. 12. Output voltage of the GMR sensor showing the variation of the inductor's value

The sensor's AC output voltages reflects the waveform of the current ripple in the inductor, which is inversely related to the value of the inductance (see equation (2)). Table 5 resumes the results presented in Fig. 12.

The measurement of the magnetic field above the inductor, and the comparison with the measurement on a reference sample makes it possible to detect a bad mounted value without the need for test points.

Table 5

Sensor output voltage according to inductor's values

\begin{tabular}{ll}
\hline \multicolumn{1}{c}{$\begin{array}{c}\text { Value of the inductance } \\
(\mu \mathrm{H})\end{array}$} & $\begin{array}{c}\text { Sensors output voltage } \\
\left(\mathrm{mV}_{\mathrm{pp}}\right)\end{array}$ \\
\hline 0,22 & 120 \\
0,47 (reference value) & 62 \\
0,82 & 38 \\
1,5 & 16 \\
\hline
\end{tabular}

\section{Conclusion and future work:}

We presented the possibility of using magnetic field signature to diagnose faulty components contactlessly on a limited physical access PCBA.

To validate the principle we used miniature near field probes and GMR sensors to measure magnetic field distributions over powered sensitive components and to give insight on the value of the component and its solder condition (soldered or non-soldered). The loading of the BUT was specifically chosen to enhance the sensitivity of the EM measurements. For the diagnosis of input decoupling capacitors and output inductor, a normal powering of the BUT was used. For the output filtering capacitors a current step was used as a stimulus to enhance the effect of these capacitors on the measured magnetic field.

These raw measurement results showed that the amplitude of the first resonance hamonic on the spectral signature acts as a sensing parameter, accurately related to the variation of the capacitor values.

Measuring the variation of the inductance value was evaluated using a commercial GMR sensor. We showed the possibility to distinguish the variation of the inductance value based on the low frequency magnetic field measured by the GMR sensor

These first experimental results demonstrate that the magnetic field probing approach can provide a viable option to detect specific component level defects and decrease the number of traditional test points while still providing access. Experiments are still on-going to validate the approach on large scale applications. A proper amplification and signal conditioning will be considered in a future work to increase measurement sensitivity and to set detection limits.

\section{Acknowledgments:}

This work was supported by the National Association of Research and Technology (ANRT) and the characterization platform of LAAS-CNRS. 


\section{References}

[1] Robinson/Verma, “Optimizing Test Strategies during PCB Design For Boards with Limited ICT Access", Proceedings of the Telecom Hardware Solutions Conference, May 2002

[2] D.Gizopoulos, Advances in ElectronicTesting: Challenges and Methodologies. New York: Springer, 2006. P.371

[3] M.N Baibich, J.M. Broto, A. Fert, F. Nguyen Van Dau, F. Petroff et al « giant magnetoresistance of (001)Fe/(001)Cr magnetic superlattice,» Phys. Rev. Lett. vol.61, no. 21, pp. 2472-2475, 1988

[4] A. Bernieri, G. Betta, L. Ferrigno, M. Laracca, "Improving performance of GMR sensors", IEEE Sensors J., vol. 13, no. 11, pp. 4513-4521, Nov. 2013.

[5] D. Cubells-Beltran, M \& Reig, Candid \& Martos, J \& Torres, Jose \& J. Soret, "Limitations of Magnetoresistive Current Sensors in Industrial Electronics Applications". INTERNATIONAL REVIEW OF ELECTRICAL ENGINEERINGIREE. 6. 423-429, 2011

[6] http://www.ti.com/lit/an/slta055/slta055.pdf 